\title{
Academic Factors Associated with College Students' Prescription Stimulant Misuse in Daily Life: An Ecological Analysis of Multiple Levels
}

\author{
Alexandra Barringer ${ }^{\mathrm{a}}$ and Lauren M. Papp ${ }^{\mathrm{a} *}$ \\ a Department of Human Development and Family Studies, University of Wisconsin-Madison, \\ Madison, WI, USA \\ *Corresponding author: \\ Lauren M. Papp \\ papp@wisc.edu \\ Department of Human Development and Family Studies \\ University of Wisconsin-Madison \\ 1300 Linden Drive \\ Madison, WI 53706 USA
}

This article has been accepted for publication in Journal of American College Health, published by Taylor \& Francis.

\section{Journal of American College Health \\ In Press, October 2020}

Funding: This work was supported by the National Institute on Drug Abuse under Grant

R01DA042093. The content is solely the responsibility of the authors and does not necessarily represent the official views of the National Institutes of Health.

Conflict of interest disclosure: The authors have no conflicts of interest to report. 


\begin{abstract}
Objective: To identify academic factors pertaining to college students, calendar timing, and particular moments that are uniquely associated with elevated likelihood of prescription stimulant misuse (intentions and actual behavior) in daily life. Participants: Participants were 297 freshmen and sophomores at a large public university in the U.S. in 2017-2019. Methods: Participants completed survey measures during lab visits and ecological momentary assessment procedures in daily life. Hypotheses were tested using multilevel models that accounted for the nested data and demographic covariates. Results: Student, calendar-based, and momentary academic factors were uniquely associated with stimulant misuse intentions in daily life. Realtime academic events, beyond the proportion of academic events experienced, emerged as a robust predictor of misuse behavior. Within-person links between real-time momentary predictors and misuse behavior were moderated by finals week timing. Conclusions: Findings offer implications for prevention and intervention strategies for college students at risk for prescription drug misuse.
\end{abstract}

Keywords: academic stress; college students; ecological momentary assessment; prescription stimulant misuse 


\section{Academic Factors Associated with College Students' Prescription Stimulant Misuse in Daily Life: An Ecological Analysis of Multiple Levels}

Prescription stimulant misuse is a recognized public health concern on college campuses. ${ }^{1,2}$ National studies reveal that young adults attending college are more likely to misuse stimulant medications relative to their non-college peers. ${ }^{3}$ Moreover, prescription stimulant misuse among college students holds serious implications for their health, including increased potential for addiction $^{4}$ and more emergency room visits. ${ }^{5}$ Research aimed at identifying reasons for the behavior overwhelmingly implicates students' goals of improving their academic performance by raising alertness ${ }^{6}$ and enhancing focus and concentration. ${ }^{7}$ Students also report misusing stimulants to make studying more enjoyable. ${ }^{8}$

Despite various academic reasons identified as a robust motivator of prescription stimulant misuse, virtually no research exists to incorporate multiple aspects of the academic context in a single study. As well, surprisingly little work has examined college students' prescription stimulant misuse in daily life, despite rigorous ecologically-based assessments of other salient substance behaviors in this developmental period, including tobacco, alcohol, and cannabis use. ${ }^{9,10}$ Guided by an ecological perspective on prescription drug misuse ${ }^{11}$ that considers multiple levels of context around the focal behavior, the current study examines academic predictors associated with college students' prescription stimulant misuse, including risk or protective factors related to individual students, calendar timing, and particular moments in daily life.

Prescription stimulant misuse is associated with individual student characteristics, including global levels of stress. ${ }^{8,12}$ Academic stress is the most commonly cited stressor among the general college student population, with approximately 50 percent of students identifying academics as being "traumatic or very difficult to handle" during the past year. ${ }^{13}$ One study 
found that college students who were more academically strained (i.e., those who viewed academic work as important yet maintained a low GPA) were more likely to engage in (pastmonth and past-year) stimulant misuse. ${ }^{14}$ However, others have reported no differences in stress levels between students who engage in the behavior and those do not. ${ }^{15,16}$ College students who appear less driven to meet academic challenges (e.g., avoid heavy coursework even when the topic is interesting) are more likely to misuse prescription stimulants. ${ }^{17}$ Prescription stimulant misuse also has been directly linked to lower GPAs. ${ }^{18,19}$

Although research on the timing of college students' prescription stimulant misuse is limited, accumulating evidence indicates temporal variation in the same population's risky alcohol use (e.g., heavier/binge drinking on holidays and during the start of the semester). ${ }^{20,21}$ Stimulant misuse behaviors also are posited to vary as a function the academic year and student schedules, although in potentially different ways than drinking. In support, data from the National Survey on Drug Use and Health (NSDUH) indicated that college students aged 18 to 22 are most likely to initiate stimulant misuse during the months of April, November, and December, ${ }^{22}$ which fall within the academic year and typically coincide with finals periods. Indeed, research has documented greater misuse of stimulant medications (assessed via both student reports and wastewater sample analysis) directly before and during final exam periods. ${ }^{23-}$

${ }^{27}$ In terms of day-to-day patterns, Greene and Maggs ${ }^{28}$ recently found that spending more time on college academics on the weekend predicted less binge drinking. These findings encourage explicit attention to the temporal context of prescription stimulant misuse and suggest that misuse behavior may be more likely to occur during more academically demanding times.

To date, prescription drug misuse has not been extensively considered in contexts of daily life. Momentary predictors of other types of substance use have been identified; for example, one 
college-based study found that heavy drinking episodes were more likely to occur when students started drinking at a private residence before moving to bars, compared to drinking at either a private residence or a bar for the entire evening. ${ }^{29}$ Another study used a prospective electronic diary-based design to identify situational influences on college students' smoking and drinking behavior, finding that students were significantly more likely to smoke while drinking if they experienced a recent increase in stress. ${ }^{30}$ Studying the focal behavior in daily life also offers the potential to examine the location of where college students tend to engage in prescription behaviors, with early qualitative findings indicating that campus libraries serve as a "hot spot" for access to diverted stimulant medications. ${ }^{31}$

In sum, while extensive previous research explicates academic factors as a leading motivation for prescription stimulant misuse, designs have not allowed for joint assessment of factors across multiple ecological levels, leaving questions about which factors are uniquely associated with prescription stimulant misuse (either intentions or behavior), and whether factors across levels might interact to predict the substance use outcomes.

\section{The current study}

The current study implemented ecological momentary assessment (EMA) to obtain contextual correlates of prescription drug misuse in daily life and capture naturalistic experiences right before misuse occurred. Our design collected reports of intention to misuse 4 classes of prescriptions along with contextual factors and hypothesized predictors in a combination of signal- and event-contingent reporting. When intention to misuse any prescription class was endorsed, a brief follow-up prompt was sent 15 minutes later and participants indicated if misuse had recently occurred. The 15-minute window was selected on the basis of its use in EMA research that identified antecedents of young adults' smoking behavior. ${ }^{32}$ The study design also 
included participants' self-reports of global measures and demographic characteristics, and the Registrar Office provided official grade data. To strengthen interpretation of the results, we included relevant covariates in the models. For example, young adults reporting stimulant misuse generally are more likely to identify as male $\mathrm{e}^{33,34}$ and White, non-Hispanic in racial/ethnic background; ${ }^{35}$ as members of fraternities and sororities, ${ }^{36}$ and have a lifetime history of medical use of prescription stimulants. ${ }^{37}$ Additionally, past work has demonstrated a positive link between experiencing adverse childhood events and engaging in stimulant misuse in adolescence. $^{38}$

The first research question was to identify academic predictors across levels (i.e., students, calendar-based timing, and particular moments) that are uniquely associated with stimulant misuse intentions and behavior in daily life. It was hypothesized that students experiencing higher levels of academic stress, as reflected by greater concern over the negative impact of their expected grades, would be more likely to report stimulant misuse intentions and behavior in daily life. We also predicted that students earning lower GPAs would be more likely to endorse misuse intentions and behavior. In terms of calendar timing, it was hypothesized that prescription stimulant misuse (intentions and behavior) would be more likely during times when academic demands were more salient, ${ }^{25}$ including during the academic year, on weekdays, and during finals periods. It was further hypothesized that momentary characteristics - specifically, when individuals were in school locations (e.g., classroom buildings, campus libraries) or experiencing academic events (such as attending class or studying) more than typical - would be positively associated with stimulant misuse intentions and behavior in daily life.

The study's novel design also permitted moderating tests to examine potential interactive effects of predictors across multiple levels. Accordingly, the second research question explored 
whether links between momentary characteristics and prescription misuse outcomes in daily life were moderated by academic calendar timing. On the basis of robust prior evidence for increased stimulant misuse during college final periods, ${ }^{27}$ it was hypothesized that the within-person associations between momentary characteristics and stimulant misuse (intentions and behavior) would be significantly stronger during finals as compared to other times.

\section{Methods}

\section{Participants}

From Fall 2017 to Fall 2019, freshmen and sophomores aged 18-21 were recruited from a university in the Midwestern United States for an ongoing longitudinal study on daily behaviors and health in college life, and oversampled for those who endorsed recent (past 3 months) misuse of a prescription medication ( $N=355$ total; 300 endorsed recent misuse). Recruitment was continuous and included mass emails, flyers across campus and the local area, and targeted ads on social media and student newsletters; all announcements mentioned the study's interest in how people use prescription medications. Prospective participants completed an online screening and a telephone call was scheduled to confirm eligibility. The present study focuses on the 300 participants deemed to have elevated risk for prescription misuse in daily life.

The screening presented 4 medication classes with common examples listed, including pain relievers, tranquilizers, stimulants, and sedatives or barbiturates. Response options were Yes/No and prospective participants could endorse misuse of multiple classes. Of the 300 elevated risk participants enrolled, most (73.3\%) endorsed recent misuse of one medication class; the remainder endorsed two or more classes. Also, the majority $(77.3 \%)$ reported recent misuse of prescription stimulants, in line with national survey data showing college students have the 
highest misuse rates for stimulants. ${ }^{3}$ Recent misuse of pain medications was endorsed by $22.3 \%$ of the sample, tranquilizers by $19.3 \%$, and sedatives or barbiturates by $11.3 \%$.

\section{Study design and procedures}

Prior to the study, university institutional review board approval and a National Institutes of Health Certificate of Confidentiality were obtained. Participants attended 2 lab sessions that were scheduled an average of 35 days apart and were trained to complete reporting procedures in daily life between the sessions. During the first session, participants completed informed consent procedures and survey measures and were trained to use an iPod Touch application designed specifically for the present research; they were provided with a private password to access the application and completed a sample report in the lab. Access to all other device features was restricted. The scheduled reporting period started the following day. Although the typical reporting period was scheduled for the 28 days following the first lab session, some participants continued reporting until they returned their device at the second lab session; reports obtained across all days were retained in the current analyses.

Consistent with published EMA protocols on addictions, ${ }^{39-41}$ both signal- and eventcontingent assessments were administered. Signal-contingent reporting involved responding to a device prompt sent within four time windows (8:00 a.m.-11:30 a.m., 11:30 a.m.-3:00 p.m., 3:00 p.m.-7:00 p.m., and 7:00 p.m.-11:00 p.m.); prompts were sent at varying times within each window across days. Participants were instructed to respond as soon as possible as appropriate. Participants were also trained to initiate an event-based report any time they intended to take a medication listed in any way a doctor did not direct them to use it. Momentary report questions focused on participants' current location and social context and potential triggers of prescription drug misuse (e.g., mood states, other substance behaviors). Signal- and event-based assessments 
were identical. A signal-contingent prompt was not sent within 2 hours after a self-initiated report had been completed to reduce burden. If misuse intention of one or more of the medication classes was endorsed, participants were sent a brief follow-up 15 minutes later ${ }^{32}$ to assess misuse behaviors that might have occurred since the completion of the associated report; participants could delay responding to the follow-up for 15 minutes. All time-stamps of report and follow-up completion were automatically recorded. During the second lab session, participants returned their devices and completed additional measures. Participants received their choice of electronic or check payments; compensation included $\$ 75$ for session 1, $\$ 84$ for reporting in daily life (prorated at $\$ 3$ per day for partial completion), $\$ 55$ for session 2, and a $\$ 36$ bonus for maintaining compliance across the reporting period.

\section{Measures}

Following Buckner and colleagues ${ }^{39}$ EMA protocol for cannabis use in daily life, participants were asked on each momentary report, "Are you about to take a medication listed here in any way a doctor did not direct you to use it? Remember, this can include using a medication without a prescription of your own; using it in greater amounts, more often, or longer than you were told to take it; or using it in any other way a doctor did not direct you to use it." Participants indicated Yes or No for 4 classes of prescription drugs (with examples provided): pain relievers, tranquilizers, stimulants, and sedatives or barbiturates. When participants endorsed one or more of the intention questions, they received a follow-up prompt 15 minutes after the associated report was completed; participants were instructed to respond to follow-ups within 15 minutes. Participants were asked, "Have you recently taken a medication listed here, in any way a doctor did not direct you to use it?" The same 4 medication classes and examples were 
presented. Analyses used dichotomous scores $(0=$ no, $1=$ yes $)$ for stimulant misuse intentions from the momentary assessments and stimulant misuse behavior from the follow-ups.

During the first lab session, participants completed a 3-item measure of academic stress, ${ }^{42}$ each rated with a Likert scale from 1 (strongly disagree) to 7 (strongly agree). A sample item was "I am worrying a great deal about the effect this semester's grades will have on my future." Item responses were averaged. The measure has shown high reliability $(\alpha=0.90)$ in previous research. ${ }^{42}$ Additionally, each participant's current-term GPA (4.0 grading scale) was obtained from the campus Registrar's Office.

Each participant's study enrollment date (based on their first lab session) was compared against the relevant campus academic calendar. A dichotomous variable indicated study participation during the academic year (fall or spring semester versus summer). Each momentary report included digital date/time stamp information that was used to create dichotomized variables reflecting whether the report was completed on a weekday (Monday through Friday versus Saturday and Sunday) and during finals period (an official study day or final exams day versus all other days).

Each momentary report prompted participants to indicate their current location from a list (e.g., home, school, work, gym). A dichotomous variable was used to indicate school location at the time of the report (school versus any other location); this was defined as any campus building, library, or study space. Each report also included a checklist of possible situations or events (e.g., academic, interpersonal, financial) the participant could be experiencing at the particular moment. In line with the current study's focus, academic event was included as a dichotomous variable (experiencing an academic event versus not). 
During the lab visit, participants also provided self-reports of sex, racial and ethnic background (recoded into non-Hispanic White versus all other groups), Greek affiliation (member versus non-member), and lifetime prescription stimulant history (participant had ever versus never received a prescription for a stimulant). Participants described their childhood family environment. ${ }^{43}$ Eleven items (e.g., "I observed quarreling or shouting between parents") were evaluated on 4-point scales from 1 (rarely or none of the time) to 4 (most or all of the time). Responses were averaged (after reverse-scoring relevant items), with higher scores reflecting more adverse family backgrounds.

\section{Data analytic strategy}

Multilevel modeling in HLM v. $8^{44}$ was employed to test the research questions; the approach draws from all participants who provided reports (whether or not they endorsed the outcome variables) and gives more weight to those with more data. The dichotomous outcome variables were estimated using hierarchical generalized linear models (HGLM) for a binary distribution. For conducting our central tests on misuse intentions and behavior outcomes, we established a significance level of $p<.025(.05 / 2)$. Additionally, we used a formula ${ }^{45}$ to convert odds ratio values to a more interpretable measure of effect size (ES) to aid in understanding the relative strength of the predictors. The analysis plan was preregistered at Open Science Framework. The analyses are based on sample sizes that exceed minimum recommendations for multilevel models. ${ }^{46}$

We conducted a 3-level HGLM to examine associations between the hypothesized academic factors and likelihood of prescription stimulant misuse intentions in daily life. Level 1 predictors included momentary school location and academic event. Level 2 predictors included finals period and day of week. We followed centering guidelines for disentangling within-person 
from between-person associations. ${ }^{47}$ Accordingly, Level 3 included person-mean variables of the momentary school location and academic event predictors along with academic year, academic stress, GPA, and the covariates. We conducted HGLM to test associations between the academic factors and misuse behavior reported on the corresponding follow-up; here we employed a 2level model given that participants did not report multiple behavior instances on the same day. Thus, Level 1 predictors included weekday, finals period, school location, and experiencing an academic event. Level 2 included person-mean variables of the momentary predictors along with academic year, academic stress, GPA, and the covariate.

To examine finals period as a moderator of the momentary predictors related to prescription stimulant misuse in daily life, we conducted two HGLMs (to estimate intentions and behavior) that included main effects of the momentary predictor and finals period and their interaction term, along with relevant covariates and person-mean scores. Significant interaction effects (evaluated at $p<.025$ ) were followed-up to probe simple slopes.

\section{Results}

\section{Preliminary results}

Two participants did not return their data collection devices, and EMA data from 1 participant was not retrievable due to a password error. The sample thus consisted of 297 participants (see Table 1). There were 23,651 momentary reports; of these, there were 478 reports in which 122 participants indicated intention to misuse a prescription stimulant. Followup prompts were completed within 30 minutes by 111 participants, yielding 305 follow-up reports from those who indicated intention to misuse any class of medication. Of these 111 participants, $70.3 \%(n=78)$ indicated that they had misused a prescription stimulant in daily life. Participants' instances of stimulant misuse behavior across the reporting period ranged from 1 to 
$11(M=2.36, S D=2.04)$. Most momentary reports $(73.3 \%)$ occurred on a weekday and $3.9 \%$ were recorded during a finals period; $13.3 \%$ were reported from a school location, and $25.4 \%$ involved an academic event.

A series of initial multilevel models examined the direct associations between several participant characteristics shown in past research to be associated with prescription misuse outcomes, including sex, race/ethnicity, Greek membership, prescription stimulant history, and childhood family adversity. In central analyses we retain those that were reliably linked ( $p$-values $<.05)$ to misuse intentions or behavior in daily life. The likelihood of intending to misuse a prescription stimulant in daily life was higher among female participants, $b=0.34, O R=1.40$, $95 \% \mathrm{CI}(1.01,1.95), p=.045$, White, non-Hispanic participants, $b=0.59, O R=1.81,95 \% \mathrm{CI}$ $(1.35,2.43), p<.001$, and those who had received a stimulant medication prescription in their lifetime, $b=0.77, O R=2.15,95 \% \mathrm{CI}(1.49,3.11), p<.001$. In contrast, the likelihood of engaging in stimulant misuse behavior in daily life was lower among participants reporting more adverse family backgrounds, $b=-0.78, O R=0.46,95 \% \mathrm{CI}(0.24,0.87), p=.018$; this finding was not consistent with the hypothesized direction. Greek membership was not associated with either outcome.

\section{Multilevel academic factors as predictors of prescription stimulant misuse}

Results from the 3-level HGLM testing the unique associations between academic factors and stimulant misuse intentions are presented in Table 2. The covariates remained significant positive predictors of misuse intentions; effect sizes were small. Controlling for these, student GPA was inversely associated with intention to misuse stimulants in daily life (small ES), whereas academic stress (i.e., worry about the negative impact of grades) was not reliably associated with misuse intentions. With respect to timing, academic year, weekday, and finals 
period each predicted greater likelihood of stimulant misuse intentions in daily life (each had a small ES). Momentary characteristics also predicted stimulant misuse intentions. Specifically, there was a positive within-person association between experiencing a real-time academic event and intending to misuse stimulants (medium ES). A significant between-person association also emerged, indicating that individuals who completed a greater proportion of their reports from a campus location were more likely, on average, to intend to misuse stimulants (medium ES).

Findings from the 2-level HGLM that estimated multiple academic factors as predictors of misuse behavior indicated that the adverse family background effect was no longer reliable ( $p$ $=.071$ ) and identified one statistically meaningful predictor (full results available from the corresponding author). Notably, there was a positive within-person association between experiencing a real-time academic event and engaging in stimulant misuse in daily life, $O R=$ $2.27,95 \%$ CI $(1.29,4.02), p=.005, d=.45$ (medium ES).

Taken together, hypothesized academic factors pertaining to students, calendar timing, and momentary situations received support as predictors of prescription stimulant misuse; results were more consistent for misuse intentions than behavior. In particular, one or more academic factors from each level tested was reliably associated with stimulant misuse intentions in daily life, with the strongest predictors obtained from momentary reports (i.e., within-person academic event and between-person location). The single reliable predictor of misuse behavior across multiple levels of analysis was experiencing a within-person momentary academic event.

\section{Moderation of real-time momentary experiences by finals period}

Results from 3-level HGLMs testing finals period as a potential moderator of the withinperson associations between real-time factors (school location, academic event) and intending to 
misuse stimulants are shown in Table 3. The interaction term did not meet the established level of significance in either model.

In 2-level HGLMs that predicted prescription stimulant misuse behavior in daily life, reliable moderation effects were documented (see Table 3). The significant interaction between school location and finals period was probed. Examination of simple slopes indicated that the within-person association between real-time school location and misuse behavior in daily life was stronger during finals periods, $O R=3.33,95 \% \mathrm{CI}(1.91,5.82), p<.001, d=.66$ (medium ES), as compared to other days of the year, $O R=0.26,95 \% \mathrm{CI}(0.04,1.58), p=.14$. In addition, follow-up tests conducted to probe the significant interaction between real-time academic event and finals period indicated that the within-person association between experiencing an academic event and misuse behavior in daily life was stronger during finals period, $O R=2.99,95 \% \mathrm{CI}$ $(1.88,4.77), p=<.001, d=.60$ (medium ES), as compared to other days, $O R=0.90,95 \% \mathrm{CI}$ $(0.24,3.48), p=.88$.

Thus, moderation hypotheses were supported in models that estimated prescription stimulant misuse behavior (but not intentions), and indicated heightened likelihood of misuse in daily life triggered by momentary academic factors during finals as compared to other times.

\section{Discussion}

The purpose of the current study was to examine academic factors associated with college students' prescription stimulant misuse across multiple levels pertaining to individuals, calendar timing, and particular moments in daily life. Based on an ecological model of prescription drug misuse in daily life, ${ }^{11}$ our design incorporated assessments of in-the-moment stimulant misuse intentions and behavior and the real-time context of misuse along with survey-based assessments and official grade reports. Recruiting a large sample of participants with elevated likelihood of 
engaging in the focal behavior was critical to ensuring that the EMA reporting approach captured stimulant misuse in daily life. Indeed, even with our approach of oversampling people who had recently misused a prescription medication (mostly stimulants), a majority of the current participants did not endorse stimulant intentions or behavior. Rigorous analytic methods included a multilevel examination of academic predictors of misuse in models with relevant control variables.

In terms of direct associations, student GPA was negatively related to misuse intentions. This is consistent with previous survey-based research. ${ }^{19,48}$ Whereas previous studies provide mixed results on academic stress levels and prescription stimulant misuse, the current study suggests that global sentiments about academic stress do not pose additional risk for stimulant misuse after accounting for other academic factors. As predicted, students were more likely to report misuse intentions during the academic year, on weekdays, and on days designated as official study or finals days. Previous work has overwhelmingly focused on lifetime or past-year occurrence of stimulant misuse, ${ }^{6}$ thus largely overlooking specific aspects of academic calendar timing. Moreover, momentary characteristics were robust predictors of stimulant misuse intentions: experiencing academic events, more than typical, predicted likelihood of reporting stimulant misuse intentions and behavior in daily life. Also, individuals completing a greater proportion of their reports from a school location (e.g., campus buildings, classrooms, libraries) were more likely, on average, to intend to misuse prescription stimulants.

Results identified reliable predictors across multiple levels of analysis of misuse intentions, while only one significant predictor (at the momentary level) emerged in the behavioral model. Discrepancies could be due to different frequencies of participants or reports, with more participants reporting intentions relative to behavior. It is also possible that there are 
meaningful differences in instances of misuse intentions that precede the behavior and those that do not (for example, whether the focal medication is available in the moment, or whether the individual is in the presence of others). We plan to undertake additional investigations to clarify these differences.

Importantly, the within-person factor of experiencing a real-time academic event, more than typical for that individual, emerged as the only reliable predictor across intentions and behavior. Additional research should examine this experience from college students' perspectives, perhaps using a qualitative approach. In a recent EMA study, Greene and Maggs ${ }^{28}$ showed that on days when students reported spending more time on their academic activities including class time, homework, and studying - they felt less positive affect and more negative affect and fatigue. While our approach relied on participants designating particular moments as involving academic (and other) events or not (and specific activities were not collected), we suspect that most academic moments consisted of the activities specified by Greene and Maggs. Further, recent work from an educational psychology perspective indicates that not all academic stress in the college setting is considered problematic. ${ }^{49}$ Moreover, our predictors focused on academic factors. Interestingly, recent research that collected college students' motivations for stimulant misuse soon after the behavior had occurred documented greater positive affect on days with the misuse. ${ }^{50}$ Clearly, incorporating multiple domains of real-time triggers of prescription drug misuse is warranted in future research.

In terms of the moderating findings, within-person links between real-time academic factors and prescription stimulant misuse varied across academic timing only for the behavioral (not the intention) model. Results indicate that factors of being in a school location (e.g., library, classroom) and experiencing an academic event (e.g., studying), more than typical for that 
individual, during finals periods are especially linked to greater likelihood of engaging in stimulant misuse. These results are consistent with research investigating stimulant medication use based on campus wastewater analysis that found greater use during academic periods marked by heightened stress,${ }^{27}$ and likely reflect greater stimulant demand and diversion in locations where students are studying during these times. We acknowledge we tested only one possible moderating factor with respect to timing (i.e., finals period). It is possible that there are other modifying contexts of timing not captured here that could be addressed in future research, such as the possibility that students may be more likely to engage in stimulant misuse during the weekend in instances when alcohol is also consumed.

\section{Limitations}

Our sample was drawn from a single large, public university in the Midwestern United States; prior work suggests meaningful differences in prescription misuse behaviors across various higher education settings. ${ }^{51}$ While generally representative of the campus community, our sample was predominately white, non-Hispanic. Future research should consider replications at other types of institutions in other regions of the country (e.g., 2-year colleges, private institutions) to clarify generalizability. Statistical power is typically lower for detecting crosslevel interaction effects than direct associations in level 1 (here, the within-person momentary links); thus, caution should be used when interpreting non-significant moderation findings from three-level models. ${ }^{52}$ Also, the findings are based on a concurrent design and thus cannot support causal interpretations. Our future investigations will be able to incorporate longitudinal followups to understand longer-term implications of prescription stimulant misuse in daily life for multiple young-adulthood health and well-being domains (e.g., academic, psychological, physical). 


\section{Implications}

Event-specific applications have been proposed as an effective way to combat situationspecific substance use on campuses, ${ }^{53}$ yet scant attention has been given to evidence-based interventions that target prescription stimulant misuse. ${ }^{54}$ The results of the current study identify targeted prevention areas within the college setting. Of note, finals week appears to be an especially high-risk window of time for prescription stimulant misuse. Among our sample of students with recent prescription drug misuse, those who report being in a campus location or engaging in academic activities, more than typical for them, during final exams are more likely to endorse stimulant misuse. To mitigate elevated prescription stimulant misuse during these periods and situations, campus-based health providers could promote healthier ways of handling stress (e.g., programs on mindfulness-based meditation). ${ }^{55}$ Students also require education about the potential serious consequences of sharing and misusing prescription stimulants. ${ }^{56}$ Providing support for study assistance or exam preparation based in college libraries and other campus locations could be valuable. Given these findings, it may be beneficial to distribute weightier assignments and exams throughout the semester to reduce stress and health risks associated with traditional finals periods. Health care providers may benefit from learning that the indication that a person had ever been prescribed a stimulant medication in their lifetime was the strongest demographic or background predictor of misuse intentions and behavior in daily life, potentially reflecting greater medication access or familiarity, and thus reinforcing the need for medication histories and ongoing monitoring. ${ }^{57}$ As well, faculty and residence advisors could be informed about the academic situations and timeframes associated with elevated stimulant misuse potential to be prepared to offer referrals to additional support. 


\section{References}

1. Bavarian N, Flay BR, Ketcham PL, Smit E. The illicit use of prescription stimulants on college campuses: A theory-guided systematic review. Health Educ Behav. 2015;42(6):719729. doi: $\underline{10.1177 / 1090198115580576}$

2. Via KD. Preventing the next epidemic: Prescribed stimulant abuse. J Nurse Pract. 2019;15(3):232-235. doi:10.1016/j.nurpra.2018.12.012

3. Schepis TS, Teter CJ, McCabe SE. Prescription drug use, misuse and related substance use disorder symptoms vary by educational status and attainment in U.S. adolescents and young adults. Drug Alcohol Depend. 2018;189:172-177. doi:10.1016/j.drugalcdep.2018.05.017

4. Swanson JM, Wigal TL, Volkow ND. Contrast of medical and nonmedical use of stimulant drugs, basis for the distinction, and risk of addiction: Comment on Smith and Farah (2011). Psychol Bull. 2011;137(5):742-748. doi:10.1037/a0024898

5. Substance Abuse and Mental Health Services Administration. The DAWN Report: Emergency Department Visits Involving Attention Deficit/Hyperactivity Disorder Stimulant Medications. Rockville: Substance Abuse and Mental Health Services Administration; 2013.

6. Prosek EA, Giordano AL, Turner KD, et al. Prevalence and correlates of stimulant medication misuse among the collegiate population. J College Stud Psychother. 2018;32(1):10-22. doi:10.1080/87568225.2017.1313691

7. Judson R, Langdon SW. Illicit use of prescription stimulants among college students: Prescription status, motives, theory of planned behaviour, knowledge and self-diagnostic tendencies. Psychol Health Med. 2009;14(1):97-104. doi:10.1080/13548500802126723 
8. Bavarian N, Flay BR, Ketcham PL, Smit E. Illicit use of prescription stimulants in a college student sample: A theory-guided analysis. Drug Alcohol Depend. 2013;132(3):665-673. doi:10.1016/j.drugalcdep.2013.04.024

9. Berg CJ, Haardörfer R, Payne JB, et al. Ecological momentary assessment of various tobacco product use among young adults. Addict Behav. 2019;92:38-46. doi:10.1016/j.addbeh.2018.12.014

10. Goodhines PA, Gellis LA, Ansell EB, Park A. Cannabis and alcohol use for sleep aid: A daily diary investigation. Health Psychol. 2019;38(11), 1036-1047. doi:10.1037/hea0000765

11. Nargiso JE, Ballard EL, Skeer MR. A systematic review of risk and protective factors associated with nonmedical use of prescription drugs among youth in the united states: A social ecological perspective. J Stud Alcohol Drugs. 2015;76(1):5-20. doi: $10.15288 /$ jsad.2015.76.5

12. Norman LB, Ford JA. Undergraduate prescription stimulant misuse and academic strain: The role of college major and graduate school plans. J Drug Issues. 2019;49(4):756-771. doi: $10.1177 / 0022042619870504$

13. American College Health Association. American College Health Association-National College Health Assessment II: Undergraduate Student Executive Summary Spring 2019. Silver Spring, MD: American College Health Association; 2019.

14. Ford JA, Schroeder RD. Academic strain and non-medical use of prescription stimulants among college students. Deviant Behav. 2009;30(1):26-53. doi:10.1080/01639620802049900 15. McNiel AD, Muzzin KB, DeWald JP, et al. The nonmedical use of prescription stimulants among dental and dental hygiene students. J Dent Educ. 2011;75(3):365-376. 
16. Wasserman JA, Fitzgerald JE, Sunny MA, Cole M, Suminski RR, Dougherty JJ. Nonmedical use of stimulants among medical students. J Am Osteopath Assoc. 2014;114(8):643-653. doi: $\underline{10.7556 / j a o a .2014 .129}$

17. Antshel KM, Parascandola T, Taylor LE, Faraone SV. Achievement goal orientation and stimulant misuse in college students. J Am Coll Health. 2019;0(0):1-9. doi: $\underline{10.1080 / 07448481.2019 .1656635}$

18. Dussault CL, Weyandt LL. An examination of prescription stimulant misuse and psychological variables among sorority and fraternity college populations. J Atten Disord. 2013;17(2):87-97.

19. McCabe SE, Teter CJ, Boyd CJ. Medical use, illicit use, and diversion of abusable prescription drugs. J Am Coll Health. 2006;54(5):269-278. doi:10.3200/JACH.54.5.269-278

20. Del Boca FK, Darkes J, Greenbaum PE, Goldman MS. Up close and personal: Temporal variability in the drinking of individual college students during their first year. J Consult Clin Psychol. 2004;72(2):155-164. doi:10.1037/0022-006X.72.2.155

21. Tremblay PF, Graham K, Wells S, Harris R, Pulford R, Roberts SE. When do first-year college students drink most during the academic year? An internet-based study of daily and weekly drinking. J Am Coll Health. 2010;58(5):401-411. doi:10.1080/07448480903540465

22. Lipari RN. Monthly Variation in Substance Use Initiation Among Full-Time College Students. Rockville: Substance Abuse and Mental Health Services Administration, Center for Behavioral Health Statistics and Quality; 2015. https://www.samhsa.gov/data/sites/default/files/report_2049/ShortReport-2049.pdf. 
23. Aikins RD. Academic performance enhancement: A qualitative study of the perceptions and habits of prescription stimulant-using college students. J Coll Stud Dev. 2011;52(5):560-576. doi: $10.1353 / \operatorname{csd} .2011 .0064$

24. Brandt SA, Taverna EC, Hallock RM. A survey of nonmedical use of tranquilizers, stimulants, and pain relievers among college students: Patterns of use among users and factors related to abstinence in non-users. Drug Alcohol Depend. 2014;143:272-276. doi:10.1016/j.drugalcdep.2014.07.034

25. Hall KM, Irwin MM, Bowman KA, Frankenberger W, Jewett DC. Illicit use of prescribed stimulant medication among college students. J Am Coll Health. 2005;53(4):167-174. doi: $\underline{10.3200 / \mathrm{JACH} \cdot 53.4 .167-174}$

26. Kerley KR, Copes H, Griffin OHI. Middle-class motives for non-medical prescription stimulant use among college students. Deviant Behav. 2015;36(7):589-603. doi: $\underline{10.1080 / 01639625.2014 .951573}$

27. Moore DR, Burgard DA, Larson RG, Ferm M. Psychostimulant use among college students during periods of high and low stress: An interdisciplinary approach utilizing both self-report and unobtrusive chemical sample data. Addict Behav. 2014;39(5):987-993. doi:10.1016/j.addbeh.2014.01.021

28. Greene KM, Maggs JL. Academic time during college: Associations with mood, tiredness, and binge drinking across days and semesters. J Adolesc. 2017;56:24-33. doi: $\underline{10.1016 / \text { j.adolescence.2016.12.001 }}$

29. Labhart F, Graham K, Wells S, Kuntsche E. Drinking before going to licensed premises: An event-level analysis of predrinking, alcohol consumption, and adverse outcomes. Alcohol Clin Exp Res. 2013;37(2):284-291. doi:10.1111/j.1530-0277.2012.01872.x 
30. Witkiewitz K, Desai SA, Steckler G, et al. Concurrent drinking and smoking among college students: An event-level analysis. Psychol Addict Behav. 2012;26(3):649-654. doi: $\underline{10.1037 / \mathrm{a} 0025363}$

31. DeSantis AD, Webb EM, Noar SM. Illicit use of prescription ADHD medications on a college campus: A multimethodological approach. J Am Coll Health. 2008;57(3):315-324. doi: $\underline{10.3200 / \mathrm{JACH} \cdot 57.3 .315-324}$

32. Thrul J, Bühler A, Ferguson SG. Situational and mood factors associated with smoking in young adult light and heavy smokers. Drug and Alcohol Rev. 2014;33(4):420-427. doi: $10.1111 /$ dar.12164

33. Faraone SV, Rostain AL, Montano CB, Mason O, Antshel KM, Newcorn JH. Systematic review: Nonmedical use of prescription stimulants: Risk factors, outcomes, and risk reduction strategies. J Am Acad Child Adolesc Psychiatry. 2020;59(1):100-112. doi: $10.1016 /$ j.jaac.2019.06.012

34. Teter CJ, McCabe SE, Cranford JA, Boyd CJ, Guthrie SK. Prevalence and motives for illicit use of prescription stimulants in an undergraduate student sample. J Am Coll Health. 2005;53(6):253-262.

35. Herman-Stahl MA, Krebs CP, Kroutil LA, Heller DC. Risk and protective factors for methamphetamine use and nonmedical use of prescription stimulants among young adults aged 18 to 25. Addict Behav. 2007;32(5):1003-1015. doi:10.1016/j.addbeh.2006.07.010

36. Benson K, Flory K, Humphreys KL, Lee SS. Misuse of stimulant medication among college students: A comprehensive review and meta-analysis. Clin Child Fam Psychol Rev. 2015;18(1):50-76. doi:10.1007/s10567-014-0177-z 
37. McCabe SE, West BT, Teter CJ, Boyd CJ. Trends in medical use, diversion, and nonmedical use of prescription medications among college students from 2003 to 2013: Connecting the dots. Addict Behav. 2014;39(7):1176-1182. doi:10.1016/j.addbeh.2014.03.008

38. Forster M, Gower AL, Borowsky IW, McMorris BJ. Associations between adverse childhood experiences, student-teacher relationships, and non-medical use of prescription medications among adolescents. Addict Behav. 2017;68:30-34. doi:10.1016/j.addbeh.2017.01.004

39. Buckner JD, Zvolensky MJ, Crosby RD, Wonderlich SA, Ecker AH, Richter A. Antecedents and consequences of cannabis use among racially diverse cannabis users: An analysis from ecological momentary assessment. Drug Alcohol Depend. 2015;147:20-25. doi:10.1016/j.drugalcdep.2014.12.022

40. Cooney NL, Litt MD, Cooney JL, Pilkey DT, Steinberg HR, Oncken CA. Alcohol and tobacco cessation in alcohol dependent smokers: Analysis of real-time reports. Psychol Addict Behav. 2007;21(3):277-286. doi:10.1037/0893-164X.21.3.277

41. Preston KL, Epstein DH. Stress in the daily lives of cocaine and heroin users: Relationship to mood, craving, relapse triggers, and cocaine use. Psychopharmacology. 2011;218(1):29-37. doi: $\underline{10.1007 / \mathrm{s} 00213-011-2183-\mathrm{x}}$

42. Macgeorge EL, Samter W, Gillihan SJ. Academic stress, supportive communication, and health. Commun Educ. 2005;54(4), 365-372. doi:10.1080/03634520500442236

43. Taylor SE, Eisenberger NI, Saxbe D, Lehman BJ, Lieberman MD. Neural responses to emotional stimuli are associated with childhood family stress. Biol Psychiatry. 2006;60(3):296-301. doi:10.1016/j.biopsych.2005.09.027 
44. Raudenbush SW, Bryk AS, Cheong YF, Congdon R. HLM 8: Hierarchical Linear and Nonlinear Modeling. Lincolnwood, IL: Scientific Software International; 2019.

45. Chinn S. A simple method for converting an odds ratio to effect size for use in meta-analysis. Stat Med. 2000;19(22):3127-3131. doi:10.1002/1097-0258(20001130)19:22<3127::AID$\underline{\mathrm{SIM} 784>3.0 . \mathrm{CO} ; 2-\mathrm{M}}$

46. Maas CJM, Hox JJ. Sufficient sample sizes for multilevel modeling. Methodology. 2005;1(3), 86-92

47. Bolger N, Laurenceau J-P. Intensive Longitudinal Methods: An Introduction to Diary and Experience Sampling Research. New York, NY: Guilford Press; 2013.

48. Arria AM, Wilcox HC, Caldeira KM, Vincent KB, Garnier-Dykstra LM, O’Grady KE. Dispelling the myth of "smart drugs": Cannabis and alcohol use problems predict nonmedical use of prescription stimulants for studying. Addict Behav. 2013;38(3), 1643-1650. doi: $\underline{10.1016 / j . a d d b e h .2012 .10 .002}$

49. Travis J, Kaszycki A, Geden M, Bunde J. Some stress is good stress: The challengehindrance framework, academic self-efficacy, and academic outcomes. [Published online ahead of print April 2, 2020]. J Educ Psychol. doi:10.1037/edu0000478

50. Schepis TS, Buckner JD, Klare DL, Wade LR, Benedetto N. Predicting college student prescription stimulant misuse: An analysis from ecological momentary assessment.

[Published online ahead of print May 27, 2020]. Exp Clin Psychopharm. doi: $10.1037 /$ pha0000386

51. McCabe SE, Knight JR, Teter CJ, Wechsler H. Non-medical use of prescription stimulants among US college students: Prevalence and correlates from a national survey. Addiction. 2005;100(1):96-106. doi:10.1111/j.1360-0443.2005.00944.x 
52. Aguinis H, Gottfredson RK, Culpepper SA. Best-practice recommendations for estimating cross-level interaction effects using multilevel modeling. J Manag Stud. 2013;39(6):14901528. doi: $\underline{10.1177 / 0149206313478188}$

53. Neighbors C, Walters ST, Lee CM, et al. Event-specific prevention: Addressing college student drinking during known windows of risk. Addict Behav. 2007;32(11):2667-2680. doi:10.1016/j.addbeh.2007.05.010

54. Weyandt LL, Oster DR, Marraccini ME, Gudmundsdottir BG, Munro BA, Rathkey ES, McCallum A. Prescription stimulant medication misuse: Where are we and where do we go from here? Exp Clin Psychopharm. 2016;24(5):400-414. doi:10.1037/pha0000093

55. Bamber MD, Schneider JK. Mindfulness-based mediation to decrease stress and anxiety in college students: A narrative synthesis of the research. Ed Res Rev. 2016;18:1-32. doi:10.1016/j.edurev.2015.12.004

56. Holt LJ, Schepis TS, Looby A, Marsh E, Marut P, Feinn R. How to say “no” most effectively: Evaluating resistance strategies for prescription stimulant diversion to inform preventive interventions. [Published online ahead of print June 26, 2019]. J Am Coll Health. doi: $\underline{10.1080 / 07448481.2019 .1626861}$

57. McCabe SE, West BT. Medical and nonmedical use of prescription stimulants: Results from a national multicohort study. J Am Acad Child Psy. 2013;52(12):1272-80.

doi:10.1016/j.jaac.2013.09.005 
Table 1. Sample characteristics, $N=297$.

\begin{tabular}{lc}
\hline & $n(\%)$ or $M \pm S D$ \\
\hline Age, years & $19.5(0.71)$ \\
Female & $205(69 \%)$ \\
Male & $92(31 \%)$ \\
Race & \\
Black or African American & $7(2.4 \%)$ \\
American Indian or Alaskan Native & $<2 \%$ \\
Asian & $24(8.1 \%)$ \\
White & $247(83.2 \%)$ \\
Native Hawaiian or other Pacific Islander & $<2 \%$ \\
Multiple races & $7(2.4 \%)$ \\
Other race & $<2 \%$ \\
Hispanic or Latino/a & $20(6.7 \%)$ \\
Greek membership & $107(36 \%)$ \\
Lifetime history of stimulant prescription & $53(17.8 \%)$ \\
Childhood family adversity & $1.50(0.44)$ \\
Academic stress & $4.90(1.67)$ \\
GPA (4.0 grading scale) & $3.45(0.62)$ \\
Academic year (study participation in Fall or Spring & $80.1 \%$ \\
semester) & \\
Momentary reports completed & $79.63(26.03)$ \\
\hline
\end{tabular}


Table 2. Multilevel model results showing the associations between academic factors and prescription stimulant misuse intentions in daily life.

\begin{tabular}{|c|c|c|c|c|}
\hline \multicolumn{5}{|l|}{ Fixed Effects } \\
\hline & $O R$ & $p$-value & $95 \% \mathrm{CI}$ & $d$ \\
\hline Intercept & 0.02 & $<.001$ & $0.02,0.03$ & -- \\
\hline \multicolumn{5}{|l|}{ Student } \\
\hline Academic stress $^{\mathrm{a}}$ & 1.03 & .06 & $0.999,1.06$ & .02 \\
\hline $\mathrm{GPA}^{\mathrm{a}}$ & 0.88 & .001 & $0.81,0.95$ & -.07 \\
\hline \multicolumn{5}{|l|}{ Calendar timing } \\
\hline Academic year & 1.21 & .008 & $1.05,1.38$ & .11 \\
\hline Weekday & 1.15 & $<.001$ & $1.09,1.21$ & .08 \\
\hline Finals period & 1.51 & $<.001$ & $1.36,1.69$ & .23 \\
\hline \multicolumn{5}{|l|}{ Momentary } \\
\hline Person-mean school location ${ }^{\mathrm{a}}$ & 3.79 & $<.001$ & $2.36,6.11$ & .74 \\
\hline Within-person school location & 1.15 & .16 & $0.89,1.02$ & .08 \\
\hline Person-mean academic event $\mathrm{t}^{\mathrm{a}}$ & 0.73 & .039 & $0.55,0.98$ & -.17 \\
\hline Within-person academic event & 1.70 & $<.001$ & $1.59,1.83$ & .29 \\
\hline \multicolumn{5}{|l|}{ Covariates } \\
\hline Gender $^{\mathrm{b}}$ & 1.21 & $<.001$ & $1.09,1.36$ & .11 \\
\hline Racial/ethnic background ${ }^{\mathrm{c}}$ & 1.31 & $<.001$ & $1.17,1.47$ & .15 \\
\hline Lifetime history of stimulant prescription & 1.57 & $<.001$ & $1.36,1.81$ & .25 \\
\hline
\end{tabular}

Note. Results based on population-average models with robust standard errors. Bolded values are statistically significant $(p<.025)$. ${ }^{\mathrm{a}}$ variable was grand-mean centered. ${ }^{\mathrm{b}}$ variable was coded $0=$ male, $1=$ female. ${ }^{c}$ variable was coded $0=$ racial/ethnic minority group member, $1=$ white. 
Table 3. Multilevel modeling results showing finals period as a moderator of within-person links between momentary experiences and prescription stimulant misuse intentions (top portion) and behavior (bottom portion) in daily life.

\begin{tabular}{|c|c|c|c|}
\hline \multicolumn{4}{|l|}{ Fixed Effects } \\
\hline \multicolumn{4}{|c|}{$\mathrm{Y}=$ Likelihood of misuse intentions } \\
\hline & OR & $p$-value & $95 \% \mathrm{CI}$ \\
\hline \multicolumn{4}{|l|}{ Model 1: School location } \\
\hline Momentary predictor & 1.27 & $<.001$ & $1.17,1.39$ \\
\hline Finals period & 1.94 & $<.001$ & $1.62,2.33$ \\
\hline Predictor $\mathrm{X}$ finals interaction & 1.44 & 0.04 & $1.02,2.03$ \\
\hline \multicolumn{4}{|l|}{ Model 2: Academic event } \\
\hline Momentary predictor & 2.14 & $<.001$ & $1.95,2.34$ \\
\hline Finals period & 1.52 & $<.001$ & $1.25,1.84$ \\
\hline Predictor X finals interaction & 1.34 & 0.05 & $1.00,1.81$ \\
\hline \multicolumn{4}{|c|}{$\mathrm{Y}=$ Likelihood of misuse behavior } \\
\hline & OR & $p$-value & $95 \% \mathrm{CI}$ \\
\hline \multicolumn{4}{|l|}{ Model 1: School location } \\
\hline Momentary predictor & 3.33 & $<.001$ & $1.91,5.82$ \\
\hline Finals period & 2.86 & .08 & $0.89,5.84$ \\
\hline Predictor X finals interaction & 0.03 & $<.001$ & $0.003,0.22$ \\
\hline \multicolumn{4}{|l|}{ Model 2: Academic event } \\
\hline Momentary predictor & 2.99 & $<.001$ & $1.88,4.77$ \\
\hline Finals period & 2.33 & .18 & $0.67,8.07$ \\
\hline Predictor X finals interaction & 0.17 & .014 & $0.04,0.69$ \\
\hline
\end{tabular}

Note. Results based on population-average models with robust standard errors. Models control for covariates and person-mean scores of the momentary predictor. Bolded values reflect statistically significant interaction terms (at $p<.025)$. 